\title{
ESPIGA
}

\section{La Escuela de} Ciencias Sociales y Humanidades en el treinta aniversario de la UNED

\author{
Lester Osorno, Xinia Zúñiga \\ y Pedro Ramírez
}

\section{RESUMEN}

El presente ensayo busca, con ocasión de los treinta años de la Universidad Estatal a Distancia, reseñar la memoria histórica de la Escuela de Ciencias Sociales y Humanidades atendiendo a los pasos más importantes, como su origen, su evolución, sus logros, sus problemas y sus principales

* Lester Osorno posee una Maestría en Administración Educativa por la Universidad La Salle de Costa Rica y es actualmente Coordinador de la Cátedra de Técnicas de Estudio a Distancia e Investigación.

Xinia Zúñiga, ex-Directora de la Escuela de Ciencias Sociales y Humanidades y Máster en Violencia Social y Familiar por la UNED

Pedro J. Ramírez, ex-director del Sistema de Estudios de Posgrado de la UNED y Doctor en Educación.

Rec. 14-7-09 Acep. 16-9-09 desafios para el futuro. El mayor desafio es y será tomar conciencia de los graves problemas sociales del país y de América Latina $y$ contribuir a sus soluciones desde una posición académica crítica e integral.

\section{PALABRAS CLAVE}

Memoria histórica, Ciencias sociales y humanidades, logros, problemas, desafios, posición crítica e integral.

\section{ABSTRACT}

As part of the thirthieth anniversary celebration of the Universidad Estatal a Distancia, this essay seeks to outline the historical memory of the School of Social Sciences and Humanities as a response to its most important steps, including its origin, evolution, achievements, problems and major challenges for the future. The biggest challenge for the School is, and will be, to become aware of the serious social problems of the country and Latin America and contribute to find solutions from a critical and comprehensive academic perspective.

\section{KEYWORDS:}

Historical memory, Social sciences and humanities, achievements, problems, challenges, critical and integral perspective.

\section{INTRODUCCIÓN}

La Universidad Estatal a Distancia fue creada en 1977 y abrió sus puertas a la población estudiantil en julio de 1980. Desde su creación y hasta 1989 la docencia se organizó en la denominada Dirección de Centros Académicos, cuya estructura administrativa se fundamentaba en la 


\section{ESPIGA}

desagregación del conocimiento en grandes áreas de estudio.

Fiel a esta idea organizativa, esta Dirección, se dividió en dos dependencias: La Oficina de Ciencias del Hombre y la Oficina de Ciencias Exactas y Naturales, a su vez estas oficinas se fraccionaron en áreas con un Coordinador de Área al frente de cada una de ellas.

La Oficina de Ciencias del Hombre estaba integrada por :

- Área de Administración y Contabilidad

- $\quad$ Área de Economía y Finanzas

- $\quad$ Área de Ciencias Sociales

- Área de Pedagogía y Humanidades y

- $\quad$ Área de Técnicas Educativas

Por su parte, la Oficina de Ciencias Exactas y Naturales por :

- $\quad$ Área de Ciencias Naturales

- Área de Matemática y

- $\quad$ Área de Tecnologías

El Coordinador de Área era el nexo entre el Jefe de Oficina y el personal bajo su mando. A cargo del Coordinador de Área se encontraban los Coordinadores de Curso, que eran los funcionarios responsables de los equipos de tutores.

Los tutores son los funcionarios directamente responsables de la entrega de la docencia.

También existía una instancia denominada Coordinación de Centros, como unidad administrativa a la que estaban adscritos los Centros Académicos y de Estudio.

La labor de tutela de los Programas Docentes o carreras se realizaba por medio de la Oficina de Programas Docentes, dependencia donde estaban ubicados los especialistas que tiene a su cargo la Dirección de los Programas (Director de carrera en las denominadas universidades convencionales). La UNED comenzó sus actividades ofreciendo los siguientes Programas Docentes:

- $\quad$ Estudios Generales

- Ciencias de la Educación con énfasis en I y II Ciclos

- Ciencias de la Educación con énfasis en Administración Educativa.

- Administración de la pequeña empresa industrial y comercial.

- Administración Pública en énfasis en Administración Bancaria. 
Luego se abrieron los Programas Docentes de:

- Administración de Empresas Agropecuarias.

- Administración de Servicios Sociales Infantiles

- Administración de Cooperativas

En la sesión n. ${ }^{\circ} 79-89$ del 8 de febrero de 1989, el Consejo Universitario de la UNED procedió a la creación de la Dirección de Docencia y con este a la de cuatro escuelas.

Históricamente, la UNED estructuró el proceso de enseñanza y aprendizaje en un proceso compuesto por fases, las cuales deben guardar una estrecha coordinación. Estas fases son: Planificación, producción, organización y entrega de la docencia. La planificación en este proceso se inicia cuando el Consejo Universitario, con base en un estudio de mercado, aprueba la apertura de un programa. En la UNED se llama programa a una carrera y la apertura de un programa o carrera constaba de dos fases: la macroprogramación y la microprogramación.

La macroprogramación se plasma en un documento donde se justifica la apertura del programa, se elabora un perfil del futuro graduado y se establece un plan de estudio que responde a ese perfil. En cambio en la microprogramación se describe cada asignatura, contenida en la macroprogramación, y se proponen los objetivos por alcanzar en cada una de ellas y se establecen los contenidos.

La siguiente fase o producción académica es aquella en la cual se elabora el paquete instructivo que consta de una unidad didáctica, una guía de estudio y ayudas didácticas como por ejemplo audiovisuales. Este proceso es guiado por una (a) productor (a) Académico.

La organización del Programa, que es la tercera fase, estaba en manos de una Oficina denominada, en ese momento, Programas Docentes, a cargo de un Coordinador de Programa, que le da al proceso una visión carrerocéntrica, es decir, la unidad necesaria para que las asignaturas que se ofrezcan respondan al perfil propuesto en la macroprogramación y no se den repeticiones de contenidos.

La última fase, denominada entrega de la docencia, estaba organizada por la Dirección de Centros Académicos, la cual, al utilizar el paquete instruccional elaborado, apoyado por tutorías, tanto presenciales, como telefónicas, llevan a cabo esta fase. Esta forma de organizar la docencia 


\section{ESPIGA}

es denominada en la UNED, visión materiocéntrica de la Docencia.

En vista de esta situación, donde los participantes no controlan algunas de las fases del proceso, el Consejo Universitario, en ese momento, toma la decisión de crear la Dirección de Docencia la cual, según el acuerdo, permitirá:

1. Obtener una coordinación más estrecha entre todas las unidades participantes en el proceso de enseñanza-aprendizaje.

2. Agilizar la toma de decisiones en la estructura organizativa responsable del proceso.

3. Establecer una única unidad de mando, responsable de todas las fases.

4. Evitar la ambigüedad que ha existido en la asignación de funciones.

5. Estimular la participación más vigorosa de los profesionales universitarios en las diferentes actividades académicas de la Universidad.

6. Promover el mejoramiento profesional del personal involucrado en los procesos de enseñanza y aprendizaje.

Crea, entonces, el Consejo Universitario, la Dirección de Docencia, como unidad de línea de la Vicerrectoría Académica, cuya labor estará orientada fundamentalmente hacia la consecución de las cuatro fases antes descritas. Esta Dirección contará con una "Oficina de Operaciones" como unidad de staff de la Dirección recién creada y cuatro Escuelas, como unidades de línea, a saber:

- Escuela de Ciencias de la Administración

- Escuela de Ciencias Exactas y Naturales

- Escuela de Ciencias Sociales y Humanidades y

- Escuela de Ciencias de la Educación

Al frente de cada Escuela se tendrá una persona con rango de Jefe de Oficina el cual evolucionará hasta convertirse en Director (a) de Escuela. Nace así la Escuela de Ciencias Sociales y Humanidades, la cual para efectos de este ensayo, será analizada y descrita en particular.

\section{Origen de la Escuela de Ciencias Sociales y Humanidades}

De la fusión del Área de Sociales con el Área de Pedagogía y Humanidades, que era la forma como estaba organizada la deno- 


\section{ESPIGA}

minada en ese momento Dirección de Centros Académicos, nació la Escuela de Ciencias Sociales y Humanidades. En sus inicios contaba con los siguientes Programas:

- $\quad$ Programa de Estudios

Generales

- $\quad$ Carrera de Estudios Universitarios y

- Carrera de Rehabilitación Profesional.

Esta Escuela, al igual que las otras, contaría a partir de entonces con profesores divididos en tres tipos o rangos, de acuerdo con las funciones que desempeña: Profesor Encargado de Programa, que tendrá a su cargo entre otras funciones:

- Coordinar el grupo de trabajo para estructurar los perfiles y componentes de los planes de estudio.

- Aportar los elementos para definir los objetivos, contenidos y actividades de los cursos.

- Coordinar el grupo de trabajo que elaborará las descripciones curriculares de los programas o carreras.

- Orientar a los estudiantes en los aspectos académicos del Programa o Carrera a su cargo.
- Organizar un banco de información relativo a los alumnos matriculados en el programa o carrera a su cargo.

El segundo tipo es el Encargado de Cátedra que tendrá como funciones las siguientes:

- Elaborar las descripciones de los cursos en coordinación con el Encargado de Programa.

- Seleccionar los materiales que se emplean para un curso.

- Decidir, si los materiales didácticos se producen con autores de la UNED o con autores externos a la Universidad.

- Determinar los medios auxiliares que se utilizarán en el paquete instructivo y que conducen a un aprendizaje eficaz.

- Verificar la validez y exactitud del contenido de los materiales que componen el paquete instructivo y en conjunto con el productor académico garantizar que la estructura, lenguaje y metodología conduzcan a un aprendizaje eficaz.

- Elaborar los instrumentos de evaluación.

Una tercera figura es el Profesor Tutor, que tiene las siguientes funciones: 


\section{ESPIGA}

- Aportar recomendaciones sobre nuevas áreas de conocimiento.

- $\quad$ Ejecutar el proceso de enseñanza-aprendizaje de acuerdo con las políticas de la cátedra.

- Colaborar en la elaboración de los instrumentos de evaluación

- Aplicar los instrumentos de evaluación

Con esta estructura inicia sus labores la Dirección de Docencia y cada una de las Escuelas que la componen así como la Oficina de Operaciones.

\section{Evolución y crecimiento de la escuela}

Para 1996 la Escuela de Ciencias Sociales y Humanidades, según Informe de Labores del Rector, contaba con los siguientes programas:

- $\quad$ Estudios Generales

- $\quad$ Estudios Universitarios

- Ciencias criminológicas

- Rehabilitación con énfasis en lo profesional

Como se puede observar, el crecimiento de la Escuela fue lento en este período de modo que solo aumenta en un Programa, el de Ciencias
Criminológicas. Sin embargo, a partir de 1997 el crecimiento es mayor. En ese año aparece un Programa más, el de Ciencias de la Educación en I y II ciclos con concentración en la enseñanza del Inglés, y en 1999 la oferta se aumenta con la Enseñanza del Francés, mediante un convenio con la Universidad de Costa Rica, y con el Programa de Música con concentración en Estudios Instrumentales, en convenio con la Sinfónica Nacional. En el año 2001, además de contar con los Programas anteriores, se inician los Programas de Teología y de Enseñanza de la Religión, en convenio con la Universidad Nacional, y un Diplomado en Ciencias Policiales. Se hace la observación de que este crecimiento no es completamente autónomo, sino sujeto a convenios con otras universidades públicas e instituciones del estado (UNED. 1996, 1997, 1999).

Para el año 2004 se concreta el traslado de la Carrera de Ciencias de la Educación con concentración en Educación Especial de la Escuela de Ciencias Sociales y Humanidades a la Escuela de Ciencias de la Educación

A partir del 2007 se entra en un proceso de mejoramiento hacia la calidad de los programas existen- 
tes y creación de nuevos programas de pre-grado, grado y posgrado. En este contexto aparecen los siguientes:

- Bachillerato y Licenciatura en Ciencias de la Educación en I y II ciclo con concentración en la enseñanza del Inglés (nuevo)

- $\quad$ Maestría en Teología (nuevo)

- Bachillerato y Lic. En ciencias policiales (en proceso)

- Bachillerato en Bibliotecología con especialización en Gestión de Bibliotecas Educativas y Centro de Recursos para el aprendizaje y la innovación (en proceso)

- Bachillerato en Bibliotecología con Especialización en Gestión de la Información y Nuevas Tecnologías (en proceso)

- Diplomado y Bachillerato en Archivística (en proceso)

- Bachillerato en Gestión y Desarrollo de la Oficina (en proceso)

- Licenciatura en Teología en Línea (en proceso)

- $\quad$ Enseñanza del Frances (en proceso)

Como se puede observar, ha sido rica la experiencia de transformación de la Escuela desde 1989 a la fecha; son 20 años de crecimiento en el número de programas, personal a cargo, actividades, apertura de cátedras nuevas, como la Manuel Mora para la difusión del pensamiento de ese gran político y jurista costarricense, la creación de la Comisión de Investigación de la Escuela con muchos proyectos de investigación en proceso o ya concluidos; también la creación de la Comisión de Extensión con múltiples proyectos que se llevan a cabo, la creación de Onda UNED, como radio educativa de la Universidad o el Programa de Género adscrito a la Escuela.

\section{Los posgrados de la Escuela de Ciencias Sociales y Humanidades: Calidad, Pertinencia y articulación}

El sistema de Estudios de Posgrado (SEP) nació en 1996, en el marco de una coyuntura internacional de expansión de la economía mundial y de una economía post-industrial impulsada por la sociedad de la información o teledirigida (Barrow, 1997; Sartori, 2000). En este marco, el conocimiento es una condición indispensable de la producción, de la competencia en el mercado y, también, del desarrollo material y humano. Como consecuencia, 


\section{ESPIGA}

la matrícula universitaria, en las últimas décadas del siglo $X X$, ha crecido en términos geométricos en todos los países, y Costa Rica no ha sido la excepción. La creación del SEP fue una respuesta a la creciente demanda de maestrías y doctorados por parte de egresados tanto de las universidades públicas como privadas.

De acuerdo con el Reglamento del SEP los programas de posgrado nacen por iniciativa de y son de las Escuelas Académicas de la Universidad. La Dirección del SEP coordina y administra los programas aprobados por las distintas instancias académicas y por CONARE. Dentro de este marco organizativo, la Escuela de Ciencias Sociales y Humanidades, inició en 1998 su oferta académica de postrados con el Programa de Maestría Profesional en Derecho Económico conénfasis en Comercio Internacional.

En la actualidad ofrece 10 programas de maestría profesional y un doctorado académico en Derecho. Dichos programas son:

- Derecho económico internacional

- Derecho del trabajo y seguridad social
- Derecho constitucional

- Criminología

- Derechos Humanos

- $\quad$ Estudio de la Violencia social y familiar

- Estudios Europeos e integración

- Teología

- Propiedad intelectual

- Iberoamericana en drogodependencia (Consorcio Iberoamericano de Universidades)

\section{Doctorados:}

- $\quad$ Derecho

Algunas reflexiones en torno a la misión y calidad de los posgrados de la Escuela.

Dar vida a 10 posgrados en un período relativamente corto (10 años) es algo meritorio para cualquier escuela universitaria, pero este hecho invita a hacer algunas consideraciones y reconsideraciones a partir del deber ser y su compromiso ético académico con la sociedad civil.

La ECSH es la Escuela con mayor número de posgrados en la UNED. En sentido estricto, su impacto debería sentirse, tanto dentro de la misma Escuela como en el resto de 
la Universidad, en las empresas y en las instituciones del Estado costarricense. ¿Por qué? Porque la creación de un Programa de Posgrado debe suponer, en una universidad pública que desarrolla 3 funciones básicas: docencia, investigación y extensión social, la fase más elevada de reflexión crítica epistemológica de parte de un equipo de académicos con la formación, experiencia, y madurez necesaria, capaz de poder percibir, identificar y diagnosticar sobre las necesidades urgentes en un área del conocimiento de la realidad natural o social y, por tanto, estar en capacidad de crear y ofrecer un programa respuesta, que produzca los cambios y las mejoras en esa área determinada de la realidad. No se puede negar que los posgrados de la Escuela han respondido a necesidades sentidas. y muchos de ellos tienen relación con las características y urgencias del desarrollo mundial y nacional. La Maestría en Derecho Económico internacional, la maestría en propiedad intelectual, la maestría en Estudios Europeos e integración son ejemplos de la acertada intencionalidad de dichos programas. Pero, ¿se justifica un programa académico con solo la buena intencionalidad? ¿Obedecen estos programas a una planificación estratégica de la Escuela y del SEP? ¿Desde una Escuela de Ciencias Sociales analítica y crítica, qué modelo de sociedad queremos? ¿Una sociedad homogeneizada y anónima en la aldea globalizada o una sociedad y estado que aspiran y luchan por tener un nombre en la historia? ¿A qué modelo responden los posgrados? ¿Son pertinentes todos los programas de posgrado de la Escuela?

Una segunda consideración consiste en señalar que la mayoría de los posgrados de la Escuela de las Ciencias Sociales y Humanidades están centrados en el área del Derecho. Se esperaría, como algo natural y lógiCo, que la ECSH tuviera entre sus programas de grado la carrera de Derecho y que desde esta fortaleza, es decir, desde la madurez académica alcanzada se proyectara a la sociedad y a las instituciones con la mayor sensibilidad social y jurídica. Sin embargo, la realidad no es así. La Escuela, desde su creación, solamente ha ofrecido cursos en diferentes áreas del derecho a distintas carreras de la Universidad. Entonces, ¿en qué se sostienen estructuralmente los planes de estudio y la gestión de los programas de posgrado en derecho? En los países anglosajones $\mathrm{y}$, en particular, en las universidades norteamericanas, la departamentalización de las áreas de conocimiento concentra y aglutina la masa crítica básica y adecuada de las carreras de 


\section{ESPIGA}

grado y posgrado. En el caso de la $\mathrm{ECSH}$, ¿qué relación tiene la cátedra de derecho con los programas de maestrías en Derecho? ¿La calidad de dichos programas no requeriría un canal de doble vía que irrigue tanto a la cátedra como a los programas de posgrado?

Un tercer aspecto por considerar es el grado de vinculación entre las maestrías en Derecho y el Doctorado en Derecho. La creación de este doctorado, en el 2000, se da en un contexto especial de la educación superior de Costa Rica. Es el primer doctorado en derecho ofertado por una universidad pública e, incluso, la Universidad de Costa Rica, con más de 60 años de ofrecer la carrera de Derecho, a la fecha aún no ofrece un doctorado tal disciplina. Entonces, se requiere que la calidad de esta especial oferta académica justificara su creación y que pasara por la vinculación estrecha entre los programas de maestrías y el programa de doctorado. De no existir esta vinculación habrá dispersión en la concepción, en los requerimientos, en la metodología y en los resultados. No conviene que los ajustes y la orientación correcta llegue en forma casual. Hay una cantidad de recursos invertidos de parte del Estado, la Universidad y los estudiantes que no deben dilapidarse al margen de una racionalidad académica, económica y ética. El manejo eficiente de los recursos se concibe actualmente como parte de una ética administrativa, de modo que el desvío de recursos de su natural propósito es como arrebatarle esos recursos a otro sector del sistema social. En este sentido, ¿ha habido planificación, control de calidad y seguimiento de la oferta de los posgrados de la Escuela? En su origen del Doctorado en Derecho se pensó que además de un excelente plan de estudios y de buenos profesores se requería una población selecta de estudiantes y que esta población provendría de las diferentes maestrías en Derecho. Era una forma de garantizar articulación, imagen, calidad y autosuficiencia económica. A la fecha, ¿se ha dado seguimiento a esta idea o son los posgrados pequeñas islas o pequeños principados monárquicos?

Una cuarta consideración está relacionada con la investigación. Una de las características de las escuelas de la universidad napoleónica fue que ponían su énfasis en la profesionalización al servicio del mercado y de los intereses del Estado. El enfoque de la universidad alemana de Humboldt hizo de las universidades centros de reflexión crítica dando un significativo impulso a 
la investigación. Hoy en la actual sociedad de la información y del conocimiento no se conciben los posgrados al margen de los centros e institutos de investigación. El conocimiento universitario que por siglos fue disciplinario, en las últimas décadas, se ha transformado en pluridisciplinario, transdisciplinario e interdisciplinario. En este nuevo modelo, la sociedad interpela a la ciencia y demanda, según cada caso, el trabajo de investigación conjunto de varias ciencias. Su legitimidad queda condicionada a la aplicación del conocimiento científico (De Souza, 2006). En este nuevo contexto, los programas de posgrados deben formar especialistas investigadores, ya que sin investigación no hay innovación y desarrollo. En esta búsqueda los investigadores forman equipos interdisciplinarios y en otros casos forman clusters de especialistas de diferentes instituciones. En muchos casos, la complejidad del objeto de estudio demanda del acercamiento epistémico de distintas especialidades y de la contribución de varias instituciones. La misión y visión de los posgrados se debe medir en función de la calidad de los aportes de las investigaciones. Por eso, en relación con los aportes a la sociedad de parte de los posgrados de la ECSH surgen las siguien- tes preguntas: quiénes investigan, qué investigan y cómo investigan. ¿Los profesores y coordinadores de los posgrados son investigadores? ¿Publican sus investigaciones en revistas nacionales e internacionales? ¿Cómo puede un programa de posgrado asegurarse que su equipo de profesores esté integrado por un porcentaje alto de investigadores? Dos vías son recomendables: una, a través de la selección, y la segunda, formando a través del programa a los recursos humanos más valiosos que luego formarán parte del staff. El qué y el cómo investigar son variables dependientes de la calidad del equipo gestor y docente de un programa.

Otro elemento por considerar son los recursos docentes y coordinadores. Hasta ahora los posgrados en la Escuela han surgido un poco a la libre, con una mezcla de buenas intenciones, intereses personales y vacíos administrativos. Como ya hemos indicado, la calidad profesional del equipo de coordinadores y docentes son la garantía de un buen posgrado. ¿Cómo asegurar la calidad tan necesaria de este equipo o qué mecanismos establecer para lograr este objetivo? CONARE para aprobar un programa exige aportar información escrita acerca del equipo de pro- 


\section{ESPIGA}

fesores y la suficiencia académica de la Escuela respectiva en relación con el personal apropiado. ¿Entonces, en dónde está la falla que impide que un programa sea efectivo en sus objetivos y metas? Porque todos sabemos que la meta de un programa de posgrado no es entregar títulos vacíos, sino formar profesionales con capacidad para innovar y para producir cambios y transformaciones en la sociedad.

Al final de estas reflexiones sobre los posgrados de la Escuela conviene enfatizar en tres aspectos para lograr calidad y una mejor reorientación hacia la excelencia académica de los mismos. La pertinencia debe de estar en la misma concepción y finalidad del programa. Responde a una justificación objetiva y a la capacidad de la institución para responder adecuadamente. Por su parte, la articulación es el mecanismo que permite amarrar todos las partes o elementos para alcanzar el producto final de calidad. En este sentido, un aspecto básico al que deben estar articulados todos los programas es la naturaleza y misión de la ECSH. Debe haber una articulación interna de cada programa y también una articulación externa, relacionada con los procesos académicos administrativos. Ambos deben complementarse e integrarse. $\mathrm{Y}$ ¿qué decir de la calidad de los recursos docentes? En una academia, a distancia o presencial, sin calidad de los recursos docentes no hay excelencia, lo que hay es autoengaño y una contribución mediocre con la sociedad. Por eso, las instituciones de educación superior con visión al futuro invierten en la formación de sus recursos. La calidad de los recursos docentes de los posgrados son la clave del éxito académico.

\section{Logros, problemas y nuevos desafíos para la escuela}

La Escuela de Ciencias Sociales y Humanidades ha pasado por diversas etapas, al igual que la Universidad. Y los logros alcanzados son productos de los diferentes actores: Directores de Escuela, Encargados de Programas, Encargados de Cátedra, profesores tutores, estudiantes y personal administrativo.

La Escuela fue considerada, a veces, en forma peyorativa, como una Escuela de servicio, como si los cursos que ofrecía tuvieran menor valía. Pero, al contrario, esta característica es una verdadera fortaleza o logro de la Escuela, por 
cuanto de esta forma se vincula a las demás Escuelas de la UNED y contribuye con la formación social y humanística tan necesarias en la formación integral de todos los estudiantes de la Universidad.

La oferta de carreras en convenio, como Música, Ciencias policiales, Teología, Educación religiosa, y Francés, abrió y enriqueció el horizonte académico de la Escuela, experiencia que después fue seguida por la creación de nuevos programas.

Otros logros importantes se pueden observar en la reforma de los Estudios Generales, en la creación de la Revista Espiga y de la Comisión de Investigación; en la apertura de nuevas Cátedras y Programas, como la carrera de Turismo, nuevos posgrados, asignación de presupuestos para proyectos de investigación y de extensión; y en la generación de políticas para el fortalecimiento de la docencia mediante el uso de plataformas tecnológicas de la información y la modernización digital.

El proyecto ONDA UNED es otro logro de la Escuela. Es el primer programa de Radio en una Unidad Académica; con la frecuencia nueva de radio y televisión este proyecto se constituye en un ante- cedente valioso para el desarrollo académico institucional.

Pero, a la par de estos logros, la Escuela tiene graves problemas que requieren de la atención de las autoridades y del esfuerzo conjunto de todos sus integrantes. Uno de ellos es la falta de espacio. Su misma naturaleza multidisciplinaria y la expansión explosiva en Programas, Cátedras, tutores y estudiantes, nuevos proyectos de investigación y de extensión demandan mayor espacio físico que permita un mejor clima organizacional, mejor servicio a estudiantes y público en general, y mejor desempeño profesional.

Otro grave problema es el agotamiento del modelo de gestión de la Universidad, que tiene su impacto en la administración y desarrollo académico de la Escuela. ¿En qué consiste este problema? Algunas aristas a continuación: a. La Escuela son muchas carreras y una gran cantidad de proyectos, que dificultan su coordinación y control, b. La labor del director queda absorbida por los trámites administrativos $\mathrm{y}$ por la larga lista de reuniones, no dejando tiempo y espacio para planear, organizar, y mejorar la vida académica de la Escuela. d. En esta misma situación se encuentran los encargados de programa y de cáte- 


\section{ESPIGA}

dra; e. Existe una concentración de poder y autoridad en las altas esferas, que limitan la capacidad de decisión a los directores de Escuela; f. El modelo administrativo está centrado en la docencia, sacrificando la investigación y la extensión. Este hecho limita la discusión académica y una serie de actividades esenciales en la academia como conferencias, simposios y congresos; g. La falta de autonomía presupuestaria retardan las tomas de decisión y hacen más compleja la administración de la Escuela.

Asociado a este problema del agotamiento del modelo de gestión surgen problemas como el del doble discurso: Por un lado está el discurso de las políticas y orientaciones que pueden ser buenas y bien intencionadas y por otro lado está la práctica, que responde a un aprendizaje en el camino, el que se encuentran obstáculos como: limitación de recursos docentes, problemas de actitud ante la academia, carga académica insuficiente para cumplir las responsabilidades con calidad, y predominio de lo tecnocrático frente a lo humanístico crítico en la formación profesional de los estudiantes.

También tenemos el problema de las modas, como la autoevaluación, la acreditación, el desarrollo de competencias, etc. El problema consiste en que se asumen sin la necesaria discusión académica, criticidad y apropiación significativa de los cambios y mejoras necesarias en la realidad y contexto institucional.

Uno de los mayores problemas es el tiempo de respuesta. El sistema se mueve a velocidad cuatrimestral como una máquina programada con plazos invariables, con sacrificio de la vida académica de los docentes, de la formación integral y crítica de los nuevos profesionales.

Todo esto obedece a un modelo que concibe la academia como entrega de la docencia, que es distinto a un espacio que genera conocimientos para las soluciones a los problemas naturales, económicos, éticos, políticos y culturales de la nación.

Otro problema es la articulación de Encargados de Programa, Encargados de Cátedra con los tutores respectivos para efecto de investigar e innovar para producir mejoras en programas, cursos y otros proyectos; el trabajo administrativo es grande y todos los componentes del sistema "académico" están obligado a responder dentro de procesos estrictos. ¿Innovaciones? Estas, sin la discusión académica crítica, 
tienen el riesgo de no calar hondo y ser perecederas

A veinte años de su creación y después de haber superado varias etapas en su crecimiento; cuáles son los nuevos desafíos que deberá enfrentar en un nuevo contexto de la educación superior y de la sociedad?

Como Escuela de Ciencias Sociales y Humanidades, su primer y principal desafío es volver sobre sus raíces ontoepistemológicas para renovar su misión de cara al país y a los grandes problemas de la sociedad global.

Otros desafíos menores que están vinculados con el primero son: a. Renovar y actualizar el Plan Estratégico que integre y articule programas de pre-grado, grado $\mathrm{y}$ posgrado, proyectos de investigación y de extensión, la programación de nuevas carreras y posgrados, la formación y capacitación de los docentes, el paso a una oferta mayor y ordenada de programas y cursos en línea, entre otros; b. Mejorar los criterios de selección de los encargados de programa, de cátedra y de los docentes en general y asegurar que el otorgamiento de la propiedad del personal académico sea solamente responsabilidad del Consejo de Escuela, porque la calidad y la excelencia académica solo puede ser buscada y valorada por los académicos, y no por los administrativos; c. Evaluar, renovar y crear nuevas unidades didácticas y materiales didácticos de modo que se garantice la actualización y la calidad de la formación de los estudiantes de todas las carreras y posgrados de la Escuela; d. Promover a nivel de la Vicerrectoría Académica un cambio del modelo de gestión académica, de modo que se priorice lo académico, una formación de alta calidad competitiva, la organización y coordinación adecuada para lograr dichas metas; e. Proponer cambios significativos en el reglamento de Cargas Académicas de modo que el profesor tutor, por una parte, alcance el trato que se merece, y los estudiantes, por otra, reciban y participen activamente en un proceso de aprendizaje significativo de una formación que los haga competir de igual a igual en un mercado laboral nacional e internacional cada vez más exigente.

\section{AMODODECONCLUSIÓN}

A treinta años de la creación de la Universidad, y a veinte de la Escuela, la sociedad y el mundo han cambiado y siguen cambiando con velocidad digital. No tomar conciencia de los hechos y no producir los cambios y mejoras necesarias es un 


\section{ESPIGA}

lamentable error, que implica costos y vida de todos los que aspiran a un título profesional para mejorar su calidad de vida y realizarse y crecer humanamente aportando como seres sociales y políticos su contribución al desarrollo integral de la sociedad costarricense.

\section{La Escuela de Ciencias Sociales y} Humanidades, más que cualquier otra Escuela, tiene una misión fundamental e indispensable en la Universidad. Es y debe ser la conciencia crítica de la Universidad, sensible más que nadie a los grandes problemas humanos y sociales de la sociedad y desde esta sensibilidad crítica y comprometida éticamente debe investigar, orientar, profesionalizar y ofrecer soluciones académicas, que por ser académicas no significa que sean retóricas y abstractas, sino juiciosamente centradas en la realidad, reflexionadas y maduras al calor de la ciencia y de la justicia social, que tiene como meta el bien común, el desarrollo en equilibrio y la paz. Esta conciencia crítica la debe hacer líder en las propuestas de cambio y de mejoras futuras en la Universidad.
BIBLIOGRAFÍA

Barrow, C. W., (1997), "La estrategia de la excelencia selectiva", en Perfiles educativos, Vol. XIX, no. 76-77.

De Souza Santos, Boaventura, (2006), La Universidad en el siglo XXI, Habana, Ed. Casa de las Américas.

Sartori, Giovanni, (2000), Homo videns, La Sociedad teledirigida, Madrid: Ed. Taurus.

Ramírez, Pedro, (2001), “Expansión y calidad de los posgrados: Estudio del modelo de la UNED" en Revista Espiga no. 4 .

Tunnermann, Carlos, (1997), La Educación Superior frente al cambio, San José: EDUCA/CSUCA.

UNED, (2006), Directrices de la UNED, San José: EUNED.

UNED (1990): Acuerdos sobre la creación de la Dirección de Docencia. San José, Publicaciones UNED.

UNED (1996; 1997; 1999; 2001; 2004; 2007 y 2008) Informes de Labores de Rectoría ante la Asamblea Universitaria, San José: EUNED,

Vargas, L.P. (1996). UNED DE COSTA RICA. Realidad y esperanza.San José: EUNED. 